\title{
RESULTS FROM IPHIR AS GUIDELINE FOR ASTEROSEISMOLOGY
}

\author{
THIERRY TOUTAIN and CLAUS FRÖHLICH
}

Physikalisch-Meteorologisches Observatorium Davos, World Radiation Center CH-7260 Davos Dorf, Switzerland

\begin{abstract}
The long uninterrupted observation of the Sun as a star with the IPHIR experiment can be used to set limits to the significance of the determination of $\mathrm{p}$-mode parameters as a function of time and the length of the time series of measurements.
\end{abstract}

\section{AMPLITUDE VARIATTION}

The detailed analysis of the long uninterrupted time series of IPHIR on the USSR PHOBOS Mission (Toutain \& Fröhlich, 1992) can be used to determine the potentiality for asteroseismology.

Results of a bi-variate spectral analysis of individual IPHIR modes with a sine wave of constant frequency and amplitude show that the mode frequency and amplitude are highly variable on time scales typical for the excitation mechanism. Examples for the amount and kind of variation of the phase and amplitude of a running fourier transform of 8 days length is illustrated in Figure 1 for $l=0$ which show the coherence ${ }^{2}$, the phase and the gain. The coherence ${ }^{2}$ times 100 corresponds to the part explained in percent in the IPHIR series with the constant sine wave. The heavy lines indicate regions with coherence ${ }^{2}>0.5$. The gain corresponds directly to the amplitude and its variation is large: it may change between two subsequent observations 5 days apart easily by a factor of 5 . There is no obvious difference in the amplitude variation between the $l=0$ and 1 mode (not shown here) indicating that interference between the unresolved modes does not cause the modulation, but the excitation-damping mechanism. This is in contrast to the conclusion of Ehgamberdiev et al. (1991) from Earthbound observations that the amplitude modulation is a combination of interference and excitation-damping with the influence of the latter being much lower than the former.

\section{FREQUENCY DETERMINATION}

In order to determine the line parameters of the modes (centroid frequency, amplitude, splitting) a model has to be used to fit the observed power spectra. The stochastic excitation suggests a Lorentzian line shape for the power defined by a centroid frequency, a linewidth, an amplitude and a noise level. Moreover, the power spectra have a $\chi^{2}$ statistics with 2 degrees of freedom yielding a very complicated power-spectrum 


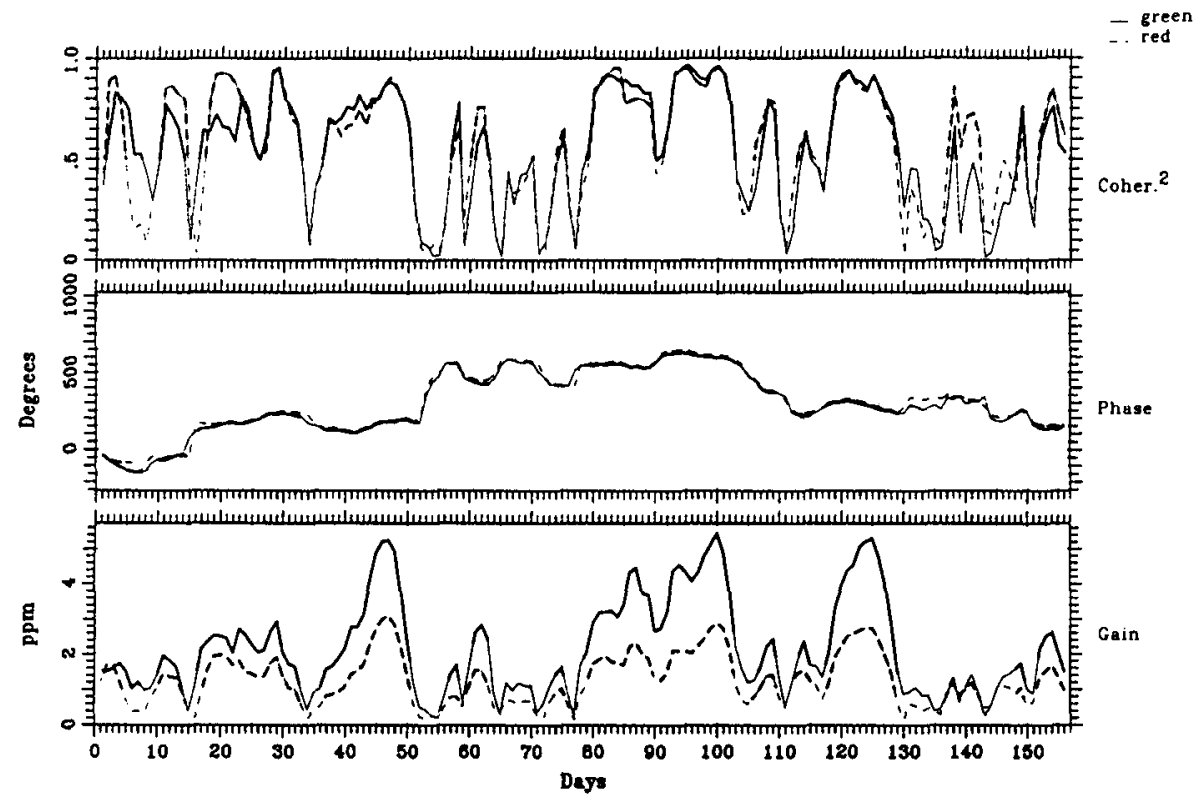

Figure 1 Bi-variate analysis of the $l=0, n=21$ mode from 8 days stretches of IPHIR data separated by one day against a sine-wave with amplitude 1ppm and the frequency found from the whole series of 160 days.

as shown in Fig. 2. In order to determine the line parameters a Lorentz line has to be fitted to the spectrum by e.g. a maximum likelihood method taking into account the proper statistics. In the case of real p-modes the situation is further complicated by the fact that lines are overlapping. Thus more than one Lorentzian has to be fitted at a time. For a doublet $l=0$ and 2 two central frequencies, a rotational splitting, two amplitudes and a noise level are fitter; for a $l=1$ mode a central frequency, a splitting, an amplitude and a noise level. For details see Toutain \& Fröhlich (1992). Because of the statistical behavior rather long series of observation are needed to determine centroid frequencies with a decent significance.

In order to study the dependence of the uncertainty on the length of time series, the IPHIR time series is analyzed using 10, 20, 40, and 80 days of observations by dividing the original one (160 days) in 16, 8, 4 and 2 series. The results of the determination of the centroid frequencies of the strongest $l=0 . .2$ lines are shown in Fig.3 with $n=21$ and 20 respectively. The shaded area is centered at the frequency determined from 160 days and the width matches the corresponding statistical uncertainty. Triangles are showing the results from Lorentzian fits, asterisks the ones of center of gravity determinations which are used if Lorentzian fits are not converging. In the case of 10 and 20 days the fitting does indeed not always converge. It works well if the initial value for the determination is close enough to the line which is in general not known a priori. In the solar case, however, the initial values are very close and the good accuracy achieved cannot be directly applied to asternseismology. One might 

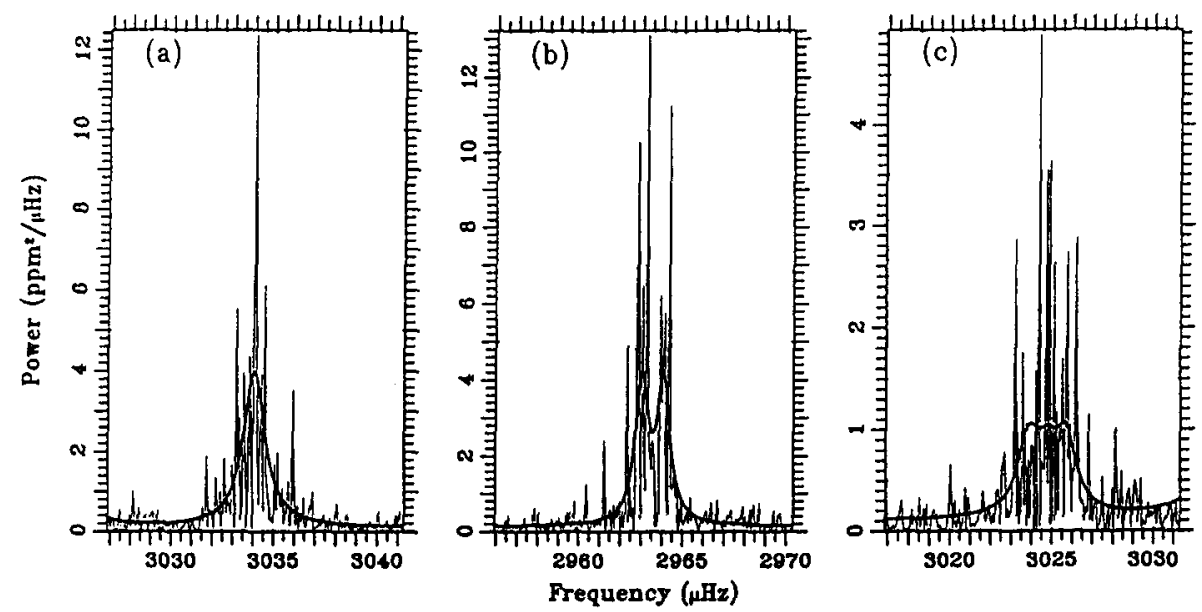

Figure 2 Examples of three p-mode lines for $l=0,1$ and 2 and $n=21,20$ and 20 respectively (from left to right) with the Lorentz lines fitted.
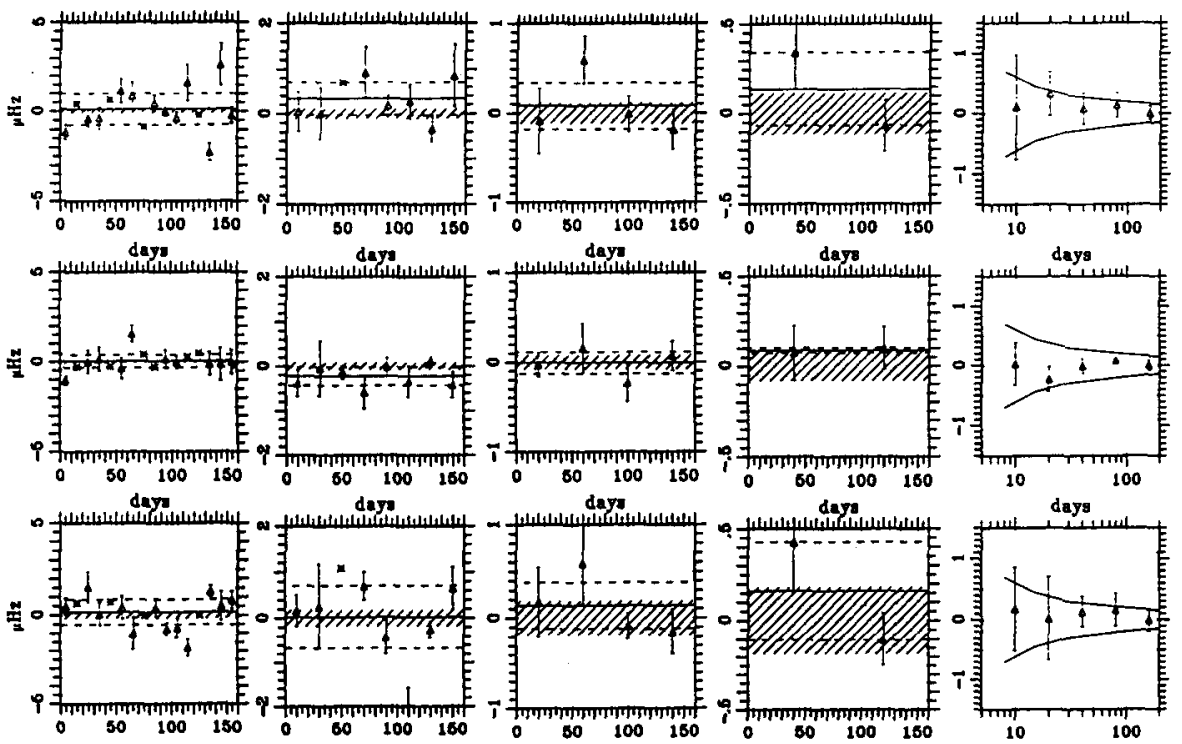

Figure 3 Determination of the centroid frequency (around $3 \mathrm{mHz}$ ) for $l=0 . . .2$ (from top to bottom) for $10,20,40$ and 80 days (from left to right). The right most column shows the uncertainty as a function of observing time.

improve the situation somehow by fixing some of the parameters such as the linewidth, but the determination would then need even more a priori knowledge. The right most column in Fig.3 summarizes the achievable uncertainties as function of observing time with the error bars indicating the range of the different determinations. 
Simulations have been performed for a $l=0$ line and 200 different $\chi^{2}$ distributions. The result of these simulations are shown as 10 limits (full lines) in the right most column of Fig.3. These indicate that the IPHIR data represent such a statistical behavior (the triangles, lie within the limits) and the uncertainty varies about as the inverse of the square root of the observation time. The influence of the method on the frequency determination is shown in Fig.4. For all three methods the starting value has been chosen as $0.5 \mu \mathrm{Hz}$ below the correct value The method of searching the center of gravity seems to depend most on the initial value used - its final value is still closer to the initial one than the true one. Thus, this method is not recommended; the two others are similar with a slight preference for maximum likelihood method.
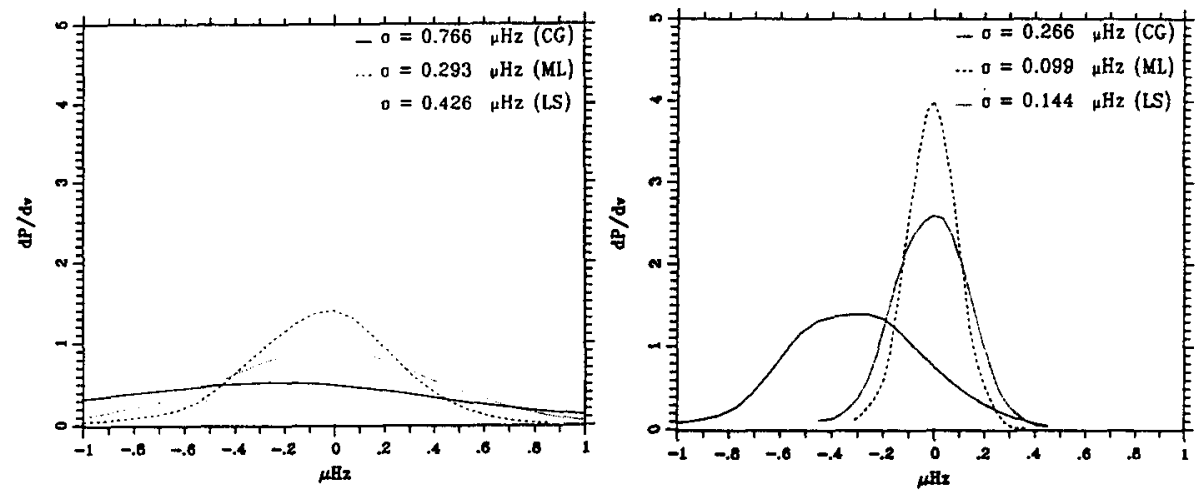

Figure 4 Distribution of the results from 1000 simulated frequency determinations from observations of 1 month (left) and 1 year (right) with three different methods: center of gravity, maximum likelihood and non-linear least squares.

\section{CONCLUSIONS}

The results of the IPHIR analysis and the simulations indicate that an accuracy of $\pm 1 \mu \mathrm{Hz}$ at the $3 \sigma$ level can only be achieved with about 3 months of observation. This is only true if the signal to noise is similar to the IPHIR lines analyzed here, namely about 30 for 6 months of observation. If it is less one might have to use even longer series. It is interesting to note, however, that the accuracy is not much dependent on $l$ although the signal to noise is quite lower for the $l=2$ line. This is probably due to the simultaneous fit with $l=0$. Again, this combined fitting may not be possible in asteroseismology as the a priori knowledge of the behavior is much lower than the one for the Sun.

\section{REFERENCES}

Ehgamberdiev, S., S.Khalikov, M.Lazrek, E.Fossat, 1991, A\&A, 253, 252.

Toutain, Th., C.Fröhlich, 1992, A\&A, 257, 287. 